\title{
Asymmetric equilibria of two nested elastic rings
}

\author{
F. Lombardo ${ }^{\mathrm{a}}$, A. Goriely ${ }^{\mathrm{b}}$, G. Napoli ${ }^{\mathrm{a}}$ \\ ${ }^{a}$ Dipartimento di Matematica e Fisica "E. De Giorgi”, Università del Salento, Lecce, Italy \\ ${ }^{b}$ Mathematical Institute, University of Oxford, Oxford, UK
}

\begin{abstract}
The packing of soft elastic structures is an important and challenging problem due to the possibility of multiple discrete and continuous zones of contact between different parts of the material. To address this problem, we consider the simplest possible packing problem of a thin elastic ring confined within another shorter flexible ring. The elastic properties as well as the dimensionality of both structures, combined with the contact condition yield a wide a variety of possible equilibrium shapes. When the rings are assumed to be inextensible and unshearable, the equilibrium shapes depend only on their relative bending stiffness $\kappa$, and on their relative length $\mu$. Whereas the symmetric equilibria for such a problem have been completely determined, the possibility of asymmetric equilibria with lower energy has not yet been considered. For a fixed value of the relative bending stiffness, we explore these symmetry-breaking equilibria as the length of the inner ring increases. We show that, for $\mu \simeq 1.9$ there is a symmetry-breaking bifurcation and asymmetric equilibria are preferred in order to relax the elastic energy.
\end{abstract}

\section{Introduction}

Many natural and man-made structures are obtained by the constrained packing of materials within a given volume. Therefore, a natural problem is to determine the morphology of confined flexible materials. This problem has many application in nature such as DNA packaging [23], mitochondria organisation [3], the morphology of plant leaves in buds [17] and even in arterial diseases $[6,26]$. Packing and folding problems play also a key role in optimization and design of flexible devices $[16,27]$. Further, beyond the elastic regime, thin sheets can develop intricate ridge networks when folded and crumpled [1]. More recently, the problem of confined elastic curves has gained interest amongst mathematicians [9, 15, 18, 7] and control engineers [2].

The confinement of a single flexible membrane or elastic sheet inside a rigid container has been studied extensively, both in two dimensions [4, 8, 24, 21, 13] and in three dimensions $[14,5]$. Whereas most of these studies assume that the container is fixed, the pressure created by the confined material can also deform the container [25, 28]. For instance microtubules surrounded by lipid membranes, can deform the membrane significantly [10]. The possibility of deforming the restraining structure creates very rich systems that can exhibit a wide variety of shapes depending on the geometric and material properties. A paradigm for such problem is the

Preprint submitted to Mechanics Research Communications problem of two nested elastic rings. In [20] we studied the existence of symmetric solutions (with respect to an axis) by increasing the length of the inner ring up to the first point of contact. The energy minimizer within the set of symmetric solutions was identified and studied as a function of the relative stiffness and length. The effect of adhesion and external pressure was also studied. However, this study did not consider the solution after the first self-contact and was restricted to symmetric solutions. The present work addresses both issues and it generalizes the analysis of $[4,24]$ by taking into account the flexibility of the container. The main result is the existence of a generic symmetry-breaking bifurcation where asymmetric shape become global minimizers of the problem. Such non-symmetric minimizers in self-contact problems are also known in other elastic problems [22, 4, 11, 19].

\section{The model}

We consider the planar equilibria of a onedimensional elastic ring constrained in another shorter elastic ring. We assume that the rings are inextensible and unshearable and neglect friction. Fixing, without loss of generality, the length of the outer ring to be one, we use the length of the inner ring $\mu$ as the main control parameter and think of an increase in length as a 
growth process. Generically, the geometry of the problem includes portions where the two rings are in contact or the inner ring touches itself. These regions evolve as the relative length changes. Thus, regions of the rings that are initially separated may end up in contact during the growth-induced packing process. We suppose that when material points are in contact they have a common position in the plane. Thus, stack of rods that are in contact in a finite region can be described by a planar elastica, endowed with a suitable bending stiffness. Consequently, the structure can be understood as a closed graph with inextensible elastic edges of unknown length. There are two kinds of nodes: (i) $Y$ - nodes where a stack of layers in contact bifurcates tangentially into two groups, and (ii) $X-$ nodes at which two rods meet at a single point (see Figure 1).

Following the notation of [12], a point $p$ on the curve can be parametrized by its Cartesian coordinates

$$
\mathbf{r}(s)=x(s) \mathbf{e}_{x}+y(s) \mathbf{e}_{y},
$$

where $s$ is the arc length. Denoting $\psi(s)$ the angle between the tangent $\tau(s)$ and the horizontal axis $\mathbf{e}_{x}$, we have

$$
\boldsymbol{\tau}(s) \equiv \mathbf{r}^{\prime}=\cos \psi(s) \mathbf{e}_{x}+\sin \psi(s) \mathbf{e}_{y},
$$

where a prime denotes the differentiation with respect to $s$. By replacing (1) into (2), we obtain:

$$
x^{\prime}=\cos \psi, \quad y^{\prime}=\sin \psi,
$$

that should be solved together with the mechanical balance equations given below.

Each rod must obey the balance of angular momentum that, in the absence of external distributed torques, reads

$$
\mathbf{m}^{\prime}(s)+\boldsymbol{\tau}(s) \times \mathbf{n}(s)=\mathbf{0},
$$

where $\mathbf{m}$ and $\mathbf{n}$ are the resultant couple and force, respectively. Moreover, the balance of linear momentum in the absence of external distributed loads assures that $\mathbf{n}$ is constant along each edge. According to the Euler-Bernoulli theory of rods, the internal moment for a planar deformation is proportional to the curvature $c=\mathrm{d} \psi / \mathrm{d} s$, so that:

$$
\mathbf{m}(s)=k c(s) \mathbf{e}_{z},
$$

where $k$ is the bending stiffness and $\mathbf{e}_{z}=\mathbf{e}_{x} \times \mathbf{e}_{y}$. We denote by $k_{-}$and $k_{+}$the bending stiffness of the inner and of the outer ring, respectively. If the edge represents a stack of rods then $k$ is the sum of the bending stiffnesses of all rods in the stack. Thus, (4) reduces to the second order ordinary differential equation

$$
k \psi^{\prime \prime}-n_{x} \sin \psi+n_{y} \cos \psi=0
$$

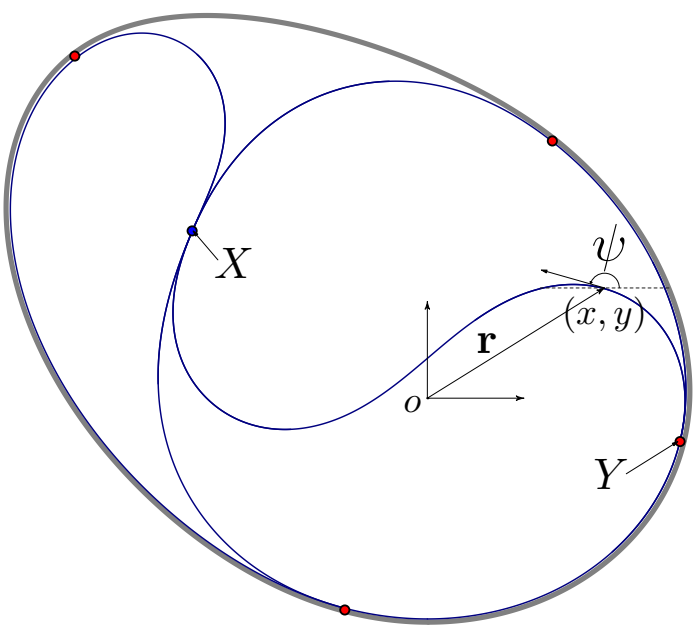

Figure 1: Schematic representation of an equilibrium shape. The $Y-$ nodes are located in correspondence of the red dot, while the $X$-node is represented by the blue dot.

where $n_{x}$ and $n_{y}$, the horizontal and vertical components of $\mathbf{n}$, are among the unknowns of the problem.

Boundary conditions are imposed at the nodes whose locations are $a$ priori unknowns. At a $Y$-node, a mother edge bifurcate into two daughter edges (each of these edges could be a single rod or a stack of rods). These groups must obey the balance [20]

$$
\begin{gathered}
\mathbf{n}+\mathbf{n}_{1}+\mathbf{n}_{2}=\mathbf{0} ; \\
\psi^{\prime}=\psi_{1}^{\prime}=\psi_{2}^{\prime},
\end{gathered}
$$

where quantities without subscript refer to the mother edge (the adhered region), while quantities with subscripts 1 and 2 refer to the daughter edges. Equation (7a) represents the balance of the force at the node, while (7b) expresses the continuity of the curvature at the detachment point. Note that (7b) implies that $x(s), y(s)$, and $\psi(s)$ are continuous at the node, for each curve.

At a $X$-node, two edges meet at an isolated point. Again, we require that each ring has continuous curvature at the node:

$$
\llbracket \psi_{1}^{\prime} \rrbracket=\llbracket \psi_{2}^{\prime} \rrbracket=0,
$$

where $\llbracket \cdot \rrbracket$ denotes the jump of a quantity at the node relative to the same rod. Further, since the contact is assumed to be frictionless the rods can freely glide on each other. This implies that the tangential component of $\mathbf{n}$ is continuous for each edge. However, the (unilateral) contact constraint induces a jump of the normal component of $\mathbf{n}$ at the node. The force conditions read:

$$
\llbracket \mathbf{n}_{1} \rrbracket \cdot \tau=\llbracket \mathbf{n}_{2} \rrbracket \cdot \tau=0,
$$




$$
\left(\llbracket \mathbf{n}_{1} \rrbracket+\llbracket \mathbf{n}_{2} \rrbracket\right) \cdot v=0,
$$

where $\boldsymbol{v}=\mathbf{e}_{z} \times \boldsymbol{\tau}$ is the normal to the curve.

Finally, we have to satisfy two global constraints: the sums of the lengths of edges belonging to the inner (resp. outer) rings must be $L_{-}$(resp. $\left.L_{+}=1\right)$, where $L_{-}$and $L_{+}$are the lengths of the inner and outer loop, respectively.

\section{Results}

We are interested in computing the shape as a function of the two dimensionless parameters

$$
\mu:=\frac{L_{-}}{L_{+}} \quad \text { and } \quad \kappa:=\frac{k_{+}}{k_{-}} .
$$

An increase in $\mu$ corresponds to a lengthening of the inner loop and in increase in $\kappa$ represents a (relative) stiffening of the outer loop. We examine the shapes for three different representative values of $\kappa: \kappa=10$ (very stiff container), $\kappa=1$ (same stiffness), and $\kappa=0.1$ (very flexible container).

We solve the problem numerically by using the Matlab routine bvp4c. For each edge of length $\ell$, there are seven unknowns $\left\{x(s), y(s), \psi(s), \psi^{\prime}(s), n_{x}, n_{y}, \ell\right\}$. The unknowns $\left\{x(s), y(s), \psi(s), \psi^{\prime}(s)\right\}$ are related to four first order differential equations: the two kinematic relations (3) and the balance of the angular momentum (6) (notice that (6) can be written as a system of two first order differential equations). The remaining three unknowns $\left\{n_{x}, n_{y}, \ell\right\}$ are constant parameters that can be determined by the boundary conditions together with the use of the global constraint on the rings length.

A simple count reveals that there are 14 boundary conditions at a $X$-node and 10 for a $Y$-node. However, the boundary conditions at the nodes are not sufficient to obtain a unique solution since the equations are invariant under rigid transformations (planar translations and rotations about $\mathbf{e}_{z}$ ). From a computational point of view, it is therefore necessary to introduce a fictitious node, that we call the fixed point. Thus, the introduction of the fixed point increases the number of edges by one. At the fixed point, we assign its position (two boundary conditions) and the orientation of the curve (one boundary condition) together with the continuity conditions (three conditions). This fixed point and the direction of the edges on which it sits remain fixed for all solutions. A further condition is necessary to avoid that the fixed point "slides" along the "broken" edge: we have required, without loss of generality, that the fixed point lies at the middle of two $Y$-nodes. For instance, in Figure 1, we have four $Y$-nodes (40 boundary conditions), one $X$-node ( 14 boundary conditions) and a fixed point ( 7 boundary conditions), which, together with the two constraints that fix the length of the rings, give 63 conditions overall. On the other hand, there are nine edges with seven unknowns for each edge which gives a total of 63 unknowns for the problem.

We start with a small value of $\mu(\simeq 1.01)$ for which the solution is known in a closed form [20] and carry a numerical continuation on $\mu$ : we use the solution obtained for a certain $\mu$ as initial condition (guess solution) of a new numerical boundary values problem where $\mu$ as been slightly increased.

For all values of $\kappa$ tested, we observe the following generic behavior: as the length of the inner loop exceeds that of the outer one $(\mu>1)$, a symmetric blister is formed. As $\mu$ increases the self-contact of the inner loop occur and finally the symmetry breaks. We can identify five stages in the folding process:

(i) For low confinements $\mu<\mu_{1}$, there is no selfcontact of the inner loop, (first column of Figure 2 ). When the outer ring is stiff, it is almost circular as the internal loop folds. On the contrary, when the outer ring is soft, it flattens in the contactless region as described in [20].

(ii) At $\mu=\mu_{1}$, a first self contact of the inner loop occurs (second column of Figure 2). For $\mu_{1}<\mu<\mu_{2}$, this self-contact remains localized at a point, while the two detachment points move on the container loop, moving away from the self-contact point. Note that the container remains almost circular in the case $\kappa=10$, while it deforms as $\kappa$ decreases.

(iii) At the critical value $\mu=\mu_{2}$, a second self-contact of the inner ring, placed on the symmetry axis, appears (third column of Figure 2). However, as $\mu$ just exceeds $\mu_{2}$ the self-contact region remains localized at a point. As $\mu$ is further increased, the second self-contact point moves along the symmetry axis, while the detachment point moves along the container walls.

(iv) For $\mu>\mu_{3}$, the contact on the bottom spreads into an extended zone of self-contact, that increases in length with $\mu$ (fourth column of Figure 2). Consequently, two symmetric lobes form. They press against each other at the self-contact point.

(v) At $\mu=\mu_{4}$, the symmetric solution becomes unstable. One of the two lobes slips under the other, breaking the symmetry and relaxing the elastic energy (last column of Figure 2). This symmetry 
$\kappa=10$

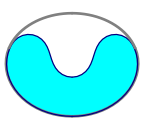

$\kappa=1$

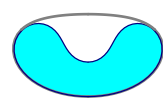

$\kappa=0.1$

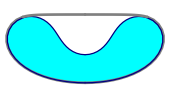

$\mu=1.10$
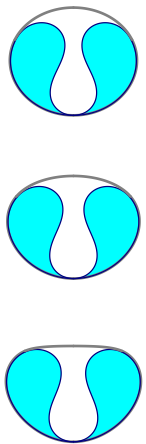

$\mu=1.50$
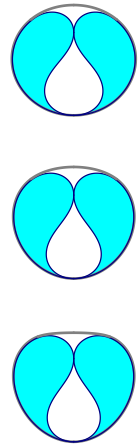

$\mu=1.67$
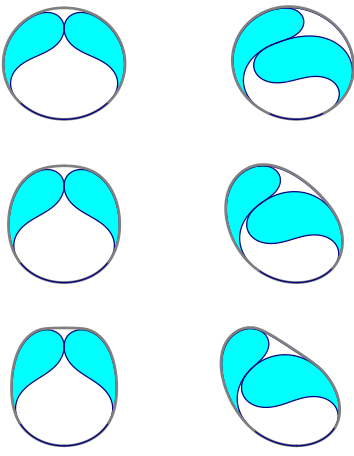

$\mu=1.88$

$\mu=2.00$

Figure 2: Equilibrium shapes as the length of the inner ring grows (from left to right) while the length of the outer loop is kept fixed. The different rows of shapes corresponds to different amounts of relative bending stiffness. The container softens from the top to the bottom.

breaking can occur more or less suddenly depending on $\kappa$.

The critical values $\mu_{i}$ for different amounts of $\kappa$ are given in Table 1. However, beyond the critical threshold $\mu_{4}$ both symmetric and non symmetric shapes exist, but the non symmetric branch has a lower total elastic energy as shown in Figure 3.

\begin{tabular}{l|llll} 
& $\mu_{1}$ & $\mu_{2}$ & $\mu_{3}$ & $\mu_{4}$ \\
\hline$\kappa=0.1$ & 1.25 & 1.66 & 1.71 & 1.88 \\
$\kappa=1$ & 1.29 & 1.64 & 1.72 & 1.90 \\
$\kappa=10$ & 1.29 & 1.62 & 1.72 & 1.95
\end{tabular}

Table 1: Critical values of length ratio $\mu$ for $\kappa=0.1,1,10$

\section{Conclusions}

The equilibrium of two elastic rings in contact provides an ideal system to explore how a thin body grows under a flexible confinement. We have extended the results of [20] in order to include self-intersections of the inner loop. The relevant parameters of this problem are the relative stiffness and the ratio of the lengths of the rings. Qualitatively, in a quasi-static growth process, the same change of morphology is observed: initially a symmetric blister forms, then the inner loop self-intersects maintaining a left-right symmetry. This symmetric state is then replaced by an asymmetric state. Note that the relative stiffness affects significantly the shape of container ring: it remains almost circular whenever the container is sufficiently hard, while it changes shape as the container softens. Furthermore, it influences the nature of the transition at the symmetry-breaking point: the transition is continuous for soft containers, whilst it occurs abruptly as the container hardens.

Acknowledgments: The support for Alain Goriely by the Engineering and Physical Sciences Research Council of Great Britain under research grant EP/R020205/1 is gratefully acknowledged.

[1] M. Ben Amar and Y. Pomeau. Crumpled paper. Proceedings of the Royal Society of London. Series A: Mathematical, Physical and Engineering Sciences, 453(1959):729, 041997.

[2] A. Blumentals. Numerical modelling of thin elastic solids in contact. Modeling and Simulation. PhD thesis, Université Grenoble Alpes, 2017.

[3] J. Bostwick, M. Miksis, and S. Davis. Elastic membranes in confinement. Journal of The Royal Society Interface, 13(120):20160408, 2016.

[4] L. Boué, M. Adda-Bedia, A. Boudaoud, D. Cassani, Y. Couder, A. Eddi, and M. Trejo. Spiral patterns in the packing of flexible structures. Phys. Rev. Lett., 97(16):166104, 102006.

[5] L. Bouzar, F. Menas, and M. M. Müller. Toroidal membrane vesicles in spherical confinement. Phys. Rev. E, 92(3):032721-, 2015.

[6] A. C. Braverman. Acute aortic dissection. Circulation, 122(2):184-188, 2010.

[7] A. Dall'Acqua and A. Pluda. Some minimization problems for planar networks of elastic curves. Geometric Flows, 2(1):105124, 2017.

[8] R. De Pascalis, G. Napoli, and S. S. Turzi. Growth-induced blisters in a circular tube. Physica D, 283:1-9, 2014.

[9] P. Dondl, L. Mugnai, and M. Röger. Confined elastic curves. SIAM Journal on Applied Mathematics, 71(6):22052226, 2018/05/03 2011.

[10] D. K. Fygenson, J. F. Marko, and A. Libchaber. Mechanics of microtubule-based membrane extension. Physical Review Letters, 79(22):4497-4500, 121997.

[11] M. Gomez, D. E. Moulton, and D. Vella. Passive control of viscous flow via elastic snap-through. Physical review letters, 119(14):144502, 2017.

[12] A. Goriely. The Mathematics and Mechanics of Biological Growth. Springer Verlag, New York, 2017.

[13] A. L. Hazel and T. Mullin. On the buckling of elastic rings by external confinement. Phil. Trans. R. Soc. A, 375(2093):20160227, 


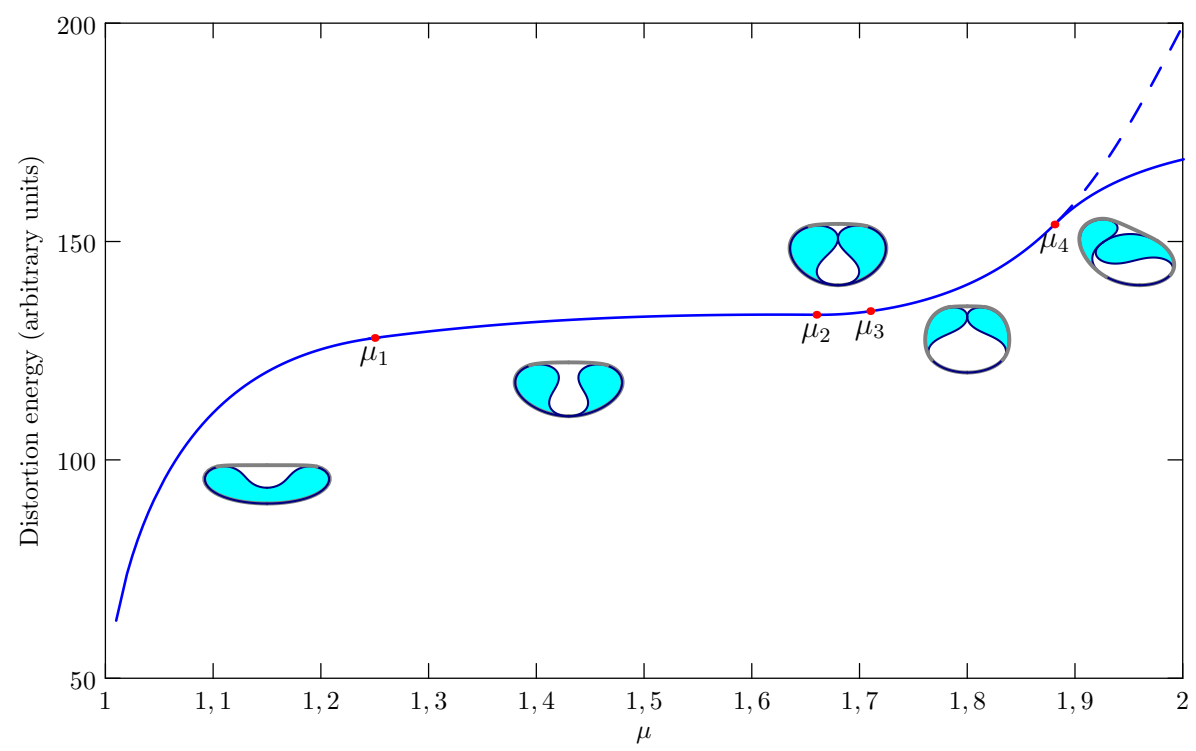

Figure 3: Total elastic energy (in arbitrary units) versus $\mu$, for $\kappa=0.1$. The critical values of $\mu_{i}(i=1,2,3,4)$ mark the beginning of a new phase of the deformation. The insets give the equilibrium shapes between two critical values. The dashed curve corresponds to the symmetric solution for $\mu>\mu_{4}$. The energy landscapes for $\kappa=1$ and $\kappa=10$ exhibit the same behavior.

2017.

[14] O. Kahraman, N. Stoop, and M. M. Müller. Morphogenesis of membrane invaginations in spherical confinement. $E P L$, 97(6):68008, 2012.

[15] T. Kemmochi. Numerical analysis of elastica with obstacle and adhesion effects. Applicable Analysis, pages 1-19, 122017.

[16] D.-H. Kim, J.-H. Ahn, W. M. Choi, H.-S. Kim, T.-H. Kim, J. Song, Y. Y. Huang, Z. Liu, C. Lu, and J. A. Rogers. Stretchable and foldable silicon integrated circuits. Science, 320(5875):507, 042008.

[17] H. Kobayashi, B. Kresling, and J. F. V. Vincent. The geometry of unfolding tree leaves. Proceedings of the Royal Society of London. Series B: Biological Sciences, 265(1391):147, 011998.

[18] T. Miura. Elastic curves and phase transitions. arXiv:1710.05890, 2017.

[19] D. E. Moulton, P. Grandgeorge, and S. Neukirch. Stable elastic knots with no self-contact. Journal of the Mechanics and Physics of Solids, 116:33-53, 2018.

[20] G. Napoli and A. Goriely. A tale of two nested elastic rings. Proceedings of the Royal Society A: Mathematical, Physical and Engineering Science, 473(2204), 082017.

[21] G. Napoli and S. Turzi. Snap buckling of a confined thin elastic sheet. Proc. R. Soc. A, 471(2183):20150444, 2015.

[22] P. Patricio, M. Adda-Bedia, and M. Ben Amar. An elastica problem: instabilities of an elastic arch. Physica-Section D, 124(1):285, 1999

[23] P. K. Purohit, M. M. Inamdar, P. D. Grayson, T. M. Squires, J. Kondev, and R. Phillips. Forces during bacteriophage dna packaging and ejection. Biophysical Journal, 88(2):851-866, 2005.

[24] J. E. Rim, P. K. Purohit, and W. S. Klug. Mechanical collapse of confined fluid membrane vesicles. Biomech. Model. Mechan., 13(6): 1277-1288, 2014.

[25] R. Vetter, F. K. Wittel, and H. J. Herrmann. Morphogenesis of filaments growing in flexible confinements. Nature Communications, 5:4437 EP -, 072014.
[26] L. Wang, N. A. Hill, S. M. Roper, and X. Luo. Modelling peeling-and pressure-driven propagation of arterial dissection. Journal of Engineering Mathematics, 109(1):227-238, 2018.

[27] C. Xiaodong. Making electrodes stretchable. Small Methods, 1(4):1600029, 2018/05/01 2017.

[28] G. Zou, X. Yi, W. Zhu, and H. Gao. Packing of flexible nanofibers in vesicles. Extreme Mechanics Letters, 19:20-26, 2018. 